\title{
Investigation of the Directional Characteristics of the Antenna of the Satellite Earth Station Using the Spiral Scanning Method
}

\author{
Andrey B. Gladyshev*a, Dmitry D. Dmitrieva, \\ Vasiliy N. Ratushniak ${ }^{a}$ and Oleg B. Gritsan ${ }^{b}$ \\ ${ }^{a}$ Siberian Federal University \\ Krasnoyarsk, Russian Federation \\ ${ }^{b}$ Reshetnev Information Satellite Systems \\ Zheleznogorsk, Russian Federation
}

Received 30.08.2021, received in revised form 02.09.2021, accepted 09.09.2021

\begin{abstract}
Results of earth station antenna experimental studies of satellite communication were shown. This antenna looks like two-mirror construction designed by Cassegrain circuit with parabolic reflector, which diameter is $1.2 \mathrm{~m}$. This results made with help of spherical scanner by spiral scanning method. Antenna directional patterns were constructed. Deviation reasons of the measured directional characteristics from the calculated ones were analyzed.
\end{abstract}

Keywords: satellite communication system, antenna parameters measurement, spherical scanner.

Citation: Gladyshev A. B., Dmitriev D. D., Ratushniak V.N., Gritsan O. B. Investigation of the directional characteristics of the antenna of the satellite earth station using the spiral scanning method, J. Sib. Fed. Univ. Eng. \& Technol., 2021, 14(7), 830-839. DOI: $10.17516 / 1999-494 X-0349$

(C) Siberian Federal University. All rights reserved

This work is licensed under a Creative Commons Attribution-Non Commercial 4.0 International License (CC BY-NC 4.0).

* Corresponding author E-mail address: a-glonass@yandex.ru 


\title{
Исследование направленных характеристик \\ антенны земной станции спутниковой связи \\ методом спирального сканирования
}

\author{
А. Б. Гладышева ${ }^{a}$ Д.Д. Дмитриев ${ }^{a}$, \\ В.Н. Ратушняк ${ }^{\text {, }}$ О.Б. Грицан ${ }^{0}$ \\ ${ }^{a}$ Сибирский федеральный университет \\ Российская Федераџия, Красноярск \\ ${ }^{\sigma}$ АО «Информационные спутниковые системы»» \\ имени академика М.Ф. Решетнёва \\ Российская Федерация, Железногорск
}

\begin{abstract}
Аннотация. В статье изложены результаты экспериментальных исследований антенны земной станции спутниковой связи, представляющей собой двухзеркальную конструкцию схемы Кассегрена с параболическим рефлектором, диаметром 1,2 м, выполненных с помощью сферического сканера методом спирального сканирования. Построены диаграммы направленности антенны, проведен анализ причин отклонения измеренных характеристик направленности от рассчитанных.
\end{abstract}

Ключевые слова: спутниковая система связи, измерение параметров антенны, сферический сканер.

Цитирование: Гладышев, А.Б. Исследование направленных характеристик антенны земной станции спутниковой связи методом спирального сканирования / А. Б. Гладышев, Д. Д. Дмитриев, В. Н. Ратушняк, О. Б. Грицан // Журн. Сиб. федер. ун-та. Техника и технологии, 2021, 14(7). C. 830-839. DOI: 10.17516/1999-494X-0349

На сегодняшний день в России отсутствует собственная полностью введенная в строй спутниковая система связи, которая бы обеспечивала непрерывное обслуживание всей территории страны [1].

Зарубежные спутниковые системы связи, которые могут предоставить свои услуги на территории нашей страны, отличаются дороговизной этих услуг. С другой стороны, ограничение возможности использования зарубежных систем спутниковой связи вызвано тем, что большая часть территории России, в частности северные регионы, находится вне зоны покрытия таких систем [2].

Таким образом, разработка и создание земных станций спутниковой связи, использующих перспективные диапазоны частот и имеющих характеристики, значительно превышающие мировые аналоги, безусловно, актуальны [3].

Характеристики станций спутниковой связи во многом зависят от параметров антенных систем, которые, в свою очередь, определяют энергетические характеристики канала связи.

В настоящее время все чаще для измерения параметров антенн применяют измерительные комплексы, основанные на измерении параметров ближнего электромагнитного поля, с последующим пересчетом в дальнее поле.

Ближнее поле антенны измеряется на поверхности, расположенной перед антенной, с использованием механизмов для перемещения измерительного зонда. Расстояние между антенной и зондом лежит в пределах от нескольких длин волн до нескольких размеров раскрыва антенны и всегда значительно меньше расстояния до границы дальней зоны. 
Используя метод ближнего поля, можно измерять такие характеристики антенных систем, как: объемные амплитудные и фазовые диаграммы направленности по основной и кроссполяризации, сечения объемных амплитудных и фазовых диаграмм направленности по основной и кросс-поляризации, уровни кросс-поляризации, поляризационные характеристики антенн, коэффициент усиления (КУ), коэффициент направленного действия (КНД), коэффициент полезного действия (КПД), шумовая температура и др. [4].

При реализации метода измерения ближнего поля наиболее универсальным способом сканирования антенн является сферическое сканирование [5].

Применяя сферический сканер в целях уменьшения времени измерений, при сохранении необходимой точности можно использовать спиральное сканирование амплитудно-фазового поля в ближней зоне антенны.

В отличие от классического сферического сканирования (рис. 1a) при спиральном сканировании точки измерений образуют собой спираль, описанную вокруг измеряемой антенны (рис. 16).

В результате данной работы был реализован алгоритм спирального сканирования на основе сканера ближнего поля фирмы Rohde \& Schwarz TS8991 [6] и построен автоматизированный измерительно-вычислительный комплекс, который обеспечивает измерение параметров апертурных антенн (зеркальные, рупорные и др.) в ближней зоне.

Данный комплекс имеет следующие технические характеристики:

1. Области сканирования:

- верхняя полусфера для сферического сканирования;

- 360 градусов по азимуту и 1000 мм по вертикали для цилиндрического сканирования.

2. Шаг между отсчетами, положение и размер области сканирования задаются оператором. При этом методика проведения измерений регламентирует выбор этих параметров оператором для получения достоверных результатов.

3. Количество частотных точек и шаг по частоте задаются оператором.

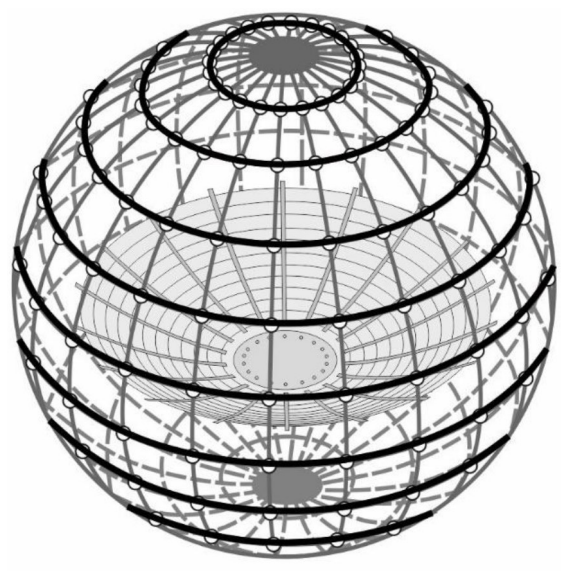

a

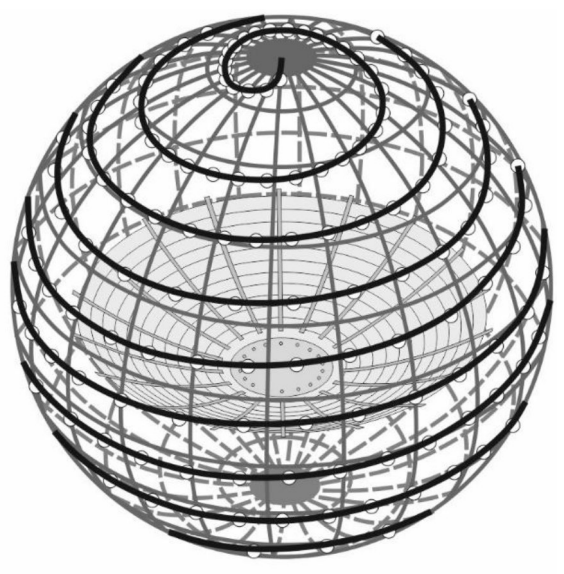

6

Рис. 1. Точки классического сферического сканирования (а) и спирального сканирования (б)

Fig.1. Scanning methods: a - Claissic spherical scanning; b - spiral scan 
4. Аппаратная погрешность измерения КУ не превышает 0,8 дБ.

Опорно-поворотное устройство (ОПУ) имеет следующие технические характеристики:

1. Масса исследуемого объекта не более 35 кг.

2. Режимы управления - ручной и от персонального компьютера.

3. Погрешность установки и индикации углов не более 0,05 градусов.

4. Отклонение от соосности посадочной поверхности антенны при вращении не более 0,5 угл. мин.

5. Отклонение от перпендикулярности и параллельности всех рабочих осей не более 0,5 угл. мин.

Схема подключения исследуемой антенны представлена на рис. 2.

Измерение амплитудно-фазового распределения (АФР) проводят с помощью векторного анализатора цепей Rohde\&Schwarz ZVA50, один порт которого подключен к излучающей антенне, а второй - к приемной, при этом система является двунаправленной.

В качестве одной из антенн используется пробник ближнего поля (зонд) на основе двухполяризационной антенны Вивальди, в качестве второй - исследуемая антенна (рис. 3).

На разработанном стенде была подвергнута испытаниям антенна земной станции спутниковой связи 08150.6220-0, представляющая собой двухзеркальную конструкцию схемы Кассегрена с параболическим рефлектором диаметром 1,2 м и контррефлектором, имеющим специальный профиль центрального сечения отражающей поверхности (рис. 4).

При измерении характеристик антенных систем необходимо соблюдать баланс между требуемой точностью и приемлемым временем измерений. В ходе проведения предварительной

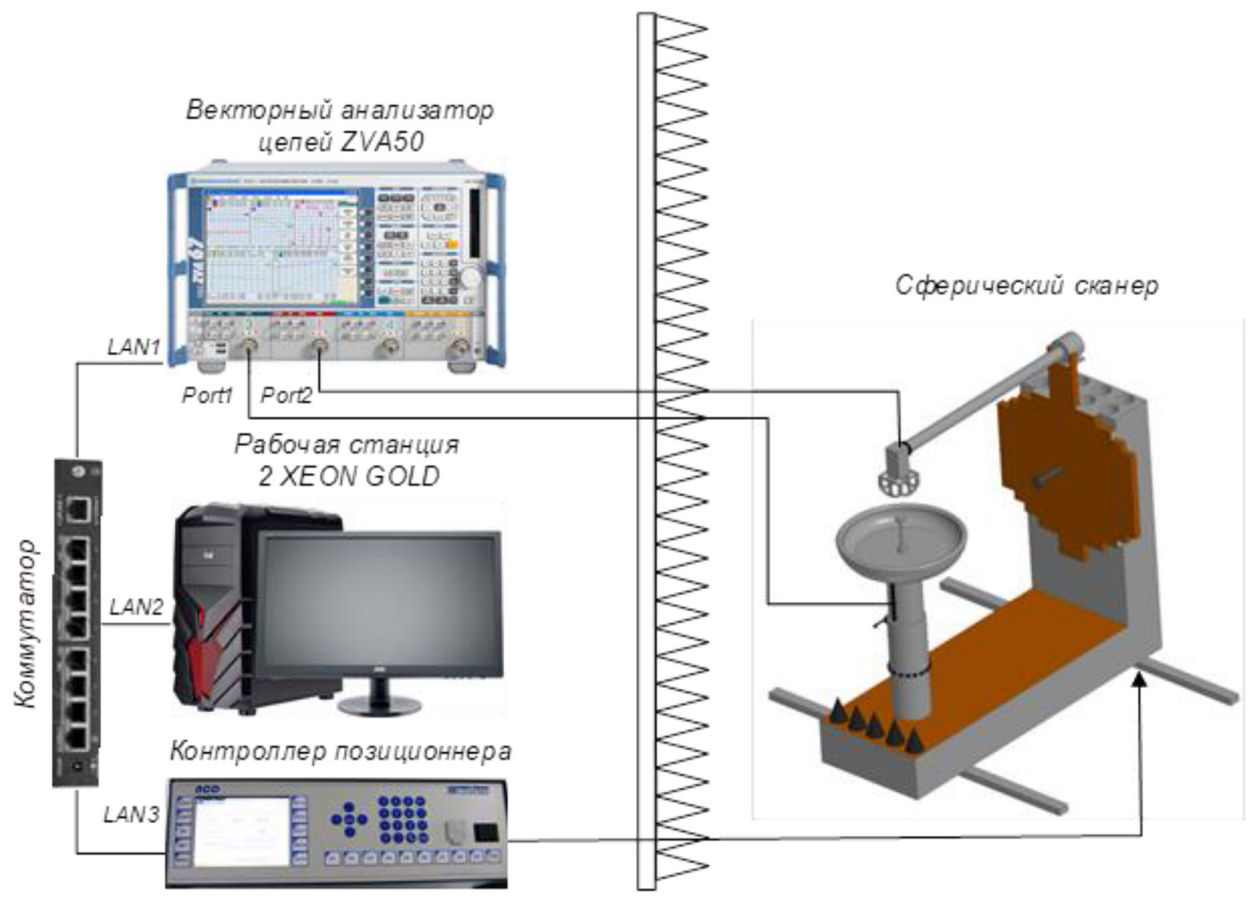

Рис. 2. Схема подключения исследуемой антенны к измерительному комплексу

Fig. 2. Connection diagram of the antenna under study to the measuring complex 


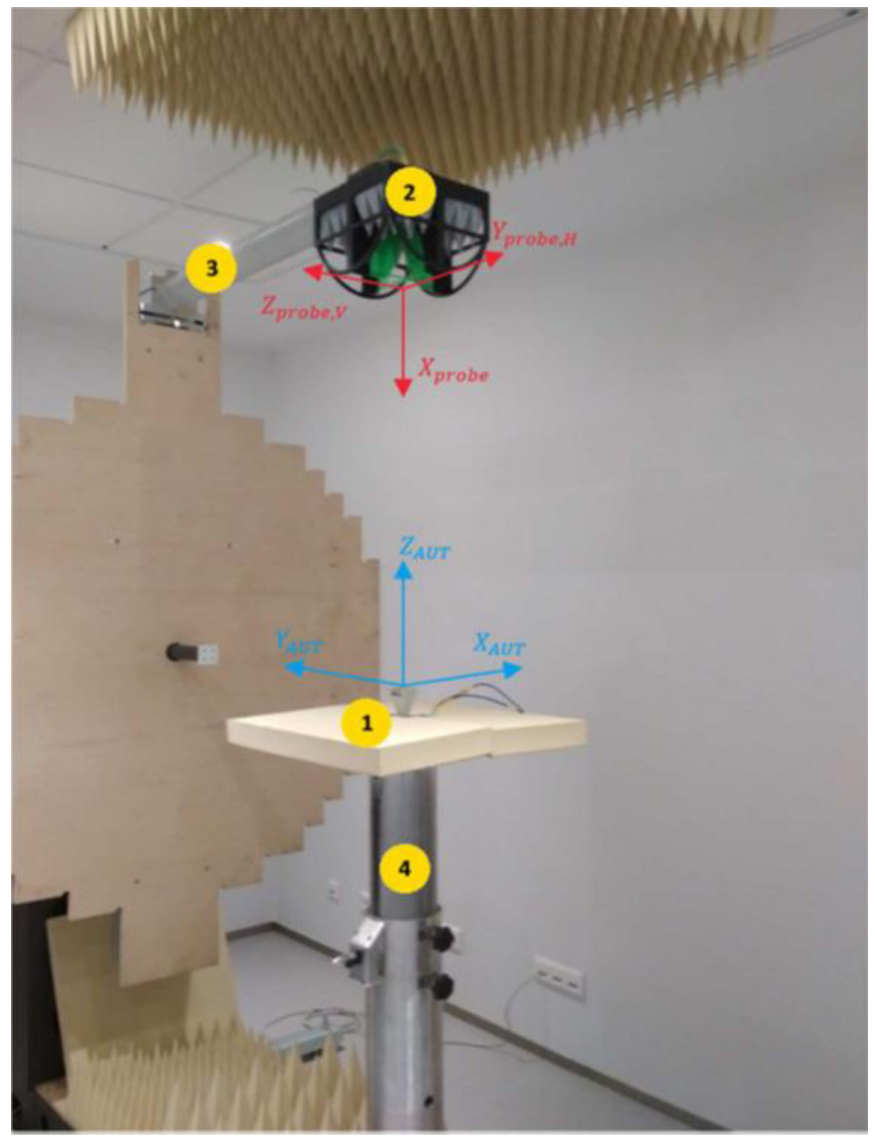

Рис. 3. Общий вид рабочей зоны сферического позиционера: 1 - эталонная (испытуемая) антенна; 2 пробник поля (зонд); 3 - мачта угломестного привода позиционера; 4 - опора стола азимутального привода позиционера

Fig. 3. General view of the working area of the spherical positioner

отработки методик измерений направленных характеристик антенн был осуществлен ряд испытаний с различным шагом измерений и углом отклонения пробника от оси антенны для определения оптимального времени измерений.

Для работы в передающем диапазоне частот была произведена стыковка антенны и анализатора цепей ZVA50 через коаксиально-волноводный переход Maury Microwave WR-22. Aнтенна была установлена на рабочий стол и больше до конца испытаний не демонтировалась.

Спиральное сканирование было организовано путем вращения поворотного стола с антенной и одновременного перемещения пробника ближнего поля в верхней полусфере.

Для пересчета ближнего поля был использован алгоритм SWE.

Испытание 1: измерение направленных характеристик антенны проводилось при максимальном отклонении пробника от оси $-8^{\circ}$ с шагом измерений $-0,2^{\circ}$. Полученные ДН на границах рабочего диапазона в главных плоскостях приведены на рис. $5 a$. Время измерений составило 1 ч 05 мин.

Испытание 2: измерение направленных характеристик антенны проводилось при максимальном отклонении пробника от оси $-16^{\circ}$ с шагом измерений $-0,2^{\circ}$. Полученные ДН на гра- 


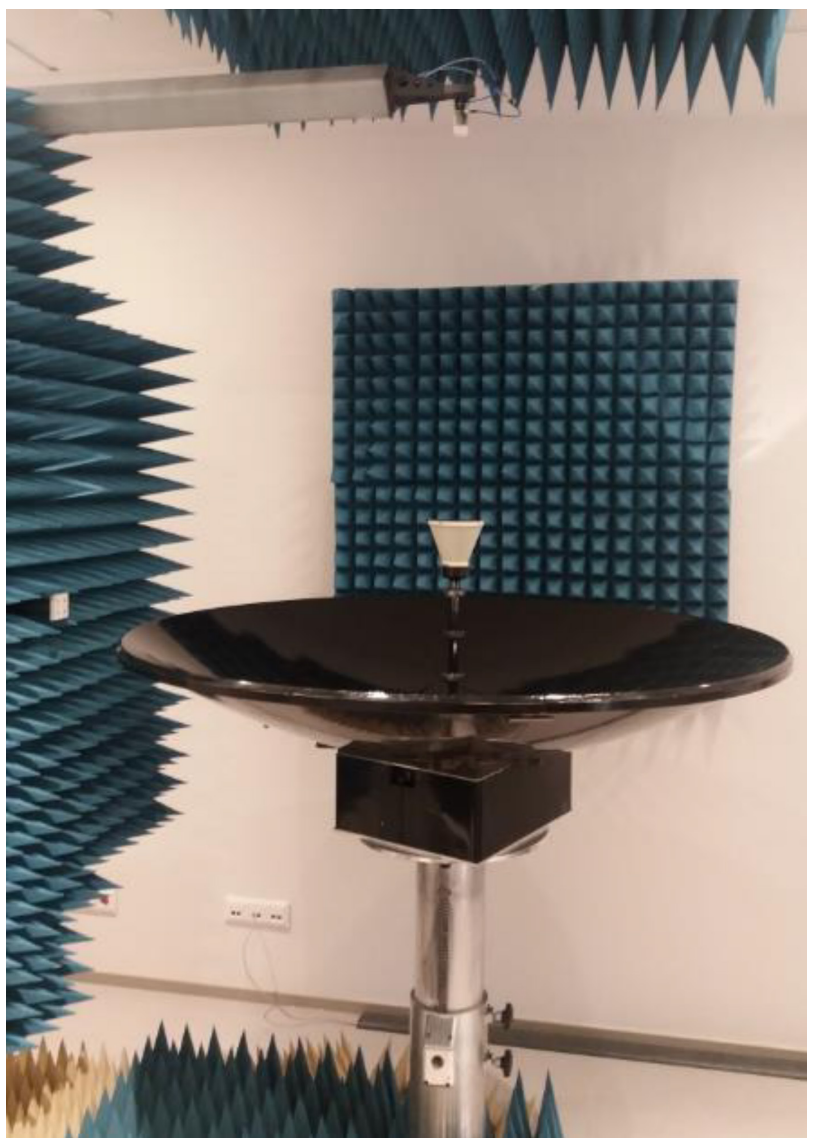

Рис. 4. Рабочее место стенда с установленной зеркальной антенной 08150.6220-0 с диаметром рефлектора $1,2 \mathrm{M}$

Fig. 4. Workplace of the stand with the installed mirror antenna $08150.6220-0$ with a reflector diameter of $1.2 \mathrm{~m}$

ницах рабочего диапазона в главных плоскостях приведены на рис 5б. Время измерений составило 2 ч 08 мин.

Испытание 3: измерение направленных характеристик антенны проводилось при максимальном отклонении пробника от оси $-18^{\circ}$ с шагом измерений $-0,3^{\circ}$. Полученные ДН на границах рабочего диапазона в главных плоскостях приведены на рис. 5в. Время измерений 1 ч 05 мин.

Испытание 4: измерение направленных характеристик антенны проводилось при максимальном отклонении пробника от оси $-24^{\circ}$ с шагом измерений $-0,3^{\circ}$. Полученные ДН на границах рабочего диапазона в главных плоскостях приведены на рис. 5 г. Время измерений 1 ч 27 мин.

Измеренные направленные характеристики исследуемой антенны представлены в табл. 1.

Результаты испытания 1 показали недостаточную точность и достоверность измерения. Это связано с тем, что при максимальном отклонении пробника от оси $-8^{\circ}$ не удается «собрать» все поле, существующее вокруг антенны. В связи с этим дальнейшие результаты, полученные в ходе испытания 1 , не исследовались. 


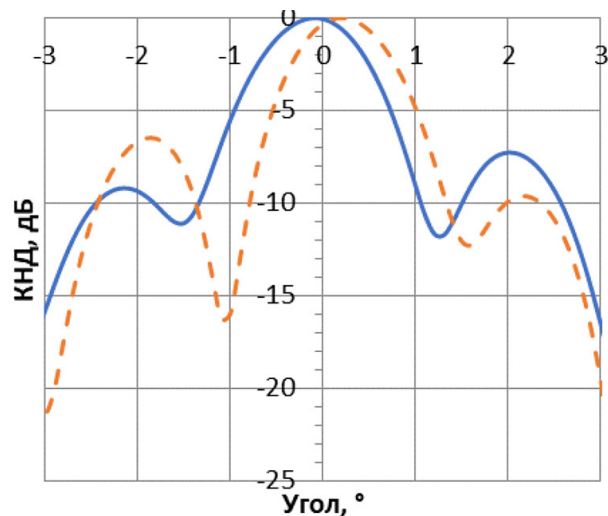

- пл. 0 - - - пл. 90

a)

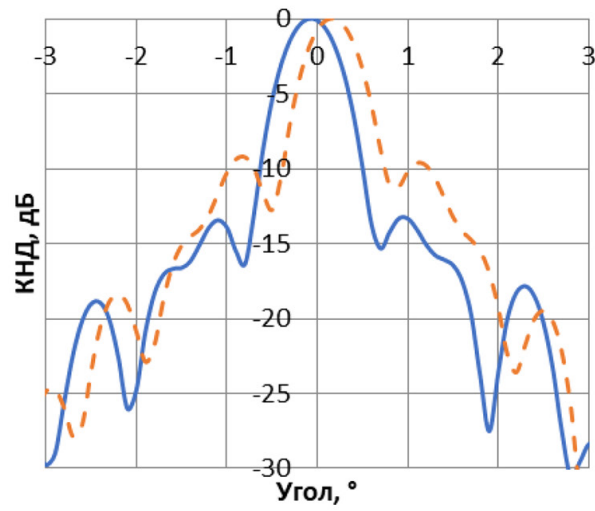

— пл. 0 - - пл. 90

в)

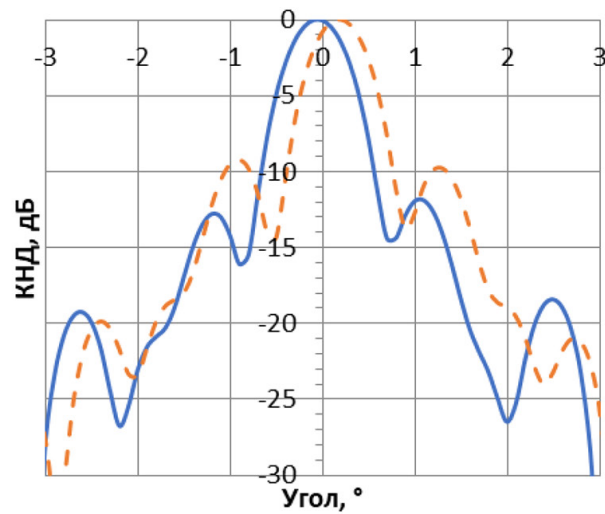

- пл $0---$ пл 90

б)

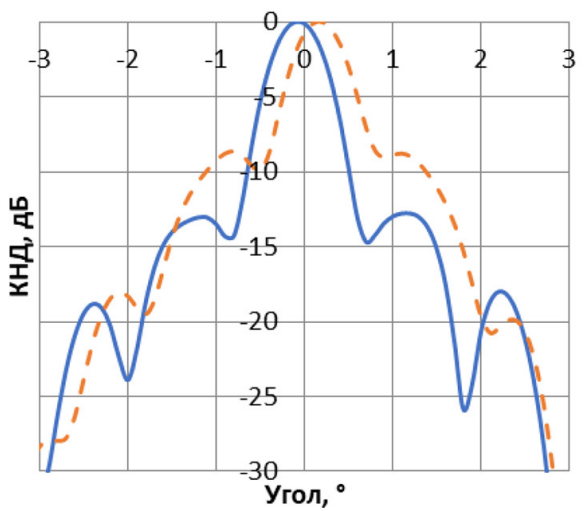

- пл. 0 - - пл. 90

г)

Рис. 5. ДН антенны на частоте 43,924 ГГц: а - испытание 1; б - испытание 2; в - испытание 3; г - испытание 4

Fig. 5. Antenna pattern at $43.924 \mathrm{GHz}$ : a - test 1 ; b - test 2 ; c - test 3 ; d - test 4

Таблица 1. Направленные характеристики антенны в плоскости $0^{\circ}$

Table 1. Directional characteristics of the antenna in the plane $0^{\circ}$

\begin{tabular}{|c|c|c|c|c|}
\hline Параметр & Исп. 1 & Исп. 2 & Исп. 3 & Исп. 4 \\
\hline Максимум КНД, дБ & 37,58 & 44,95 & 45,54 & 44,84 \\
\hline $\begin{array}{l}\text { Направление главного } \\
\text { максимума, }^{\circ}\end{array}$ & минус 0,1 & минус 0,1 & минус 0,1 & минус 0,1 \\
\hline УБЛ, дБ & минус 7,28 & минус 11,86 & минус 14,23 & минус 12,8 \\
\hline ширина ДН, ${ }^{\circ}$ & 1,4 & 0,7 & 0,65 & 0,65 \\
\hline КУ, дБ & 16,77 & 32,33 & 33,86 & 33,65 \\
\hline Время измерений, мин & 65 & 128 & 65 & 87 \\
\hline
\end{tabular}


По результатам испытаний 2-4 были получены диаграммы направленности исследуемой зеркальной антенны для углов отклонения пробника от оси соответственно 16, 18, и 24 градуса для плоскостей $0^{\circ}$ и $90^{\circ}$. Данные диаграммы направленности представлены на рис. 6 .

По результатам испытаний 2-4 можно сделать вывод, что измерения с шагом $0,2^{\circ}$ более точны в связи с тем, что измеряется большее количество точек, однако обработка таких измерений требует существенно больше времени. При этом значения КНД в максимуме для всех измерений находятся в пределах погрешности измерений ( $\pm 0,8$ дБ), а наибольшее несоответствие наблюдается при определении уровня первого бокового лепестка - более 2 дБ.

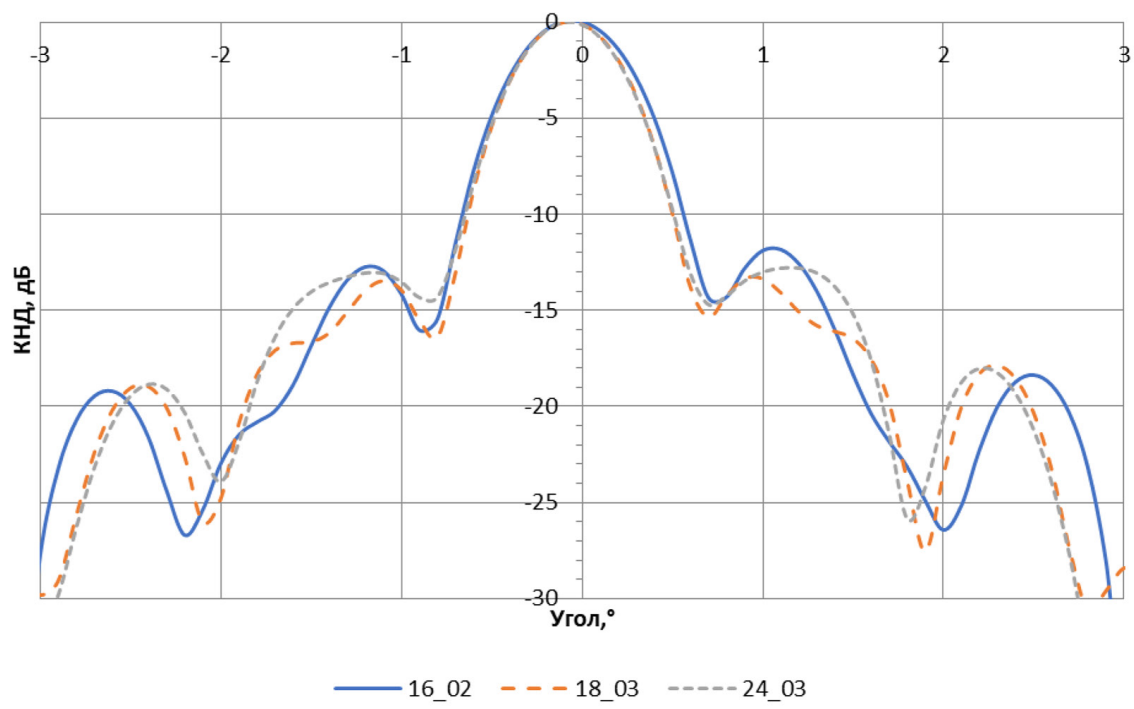

a)

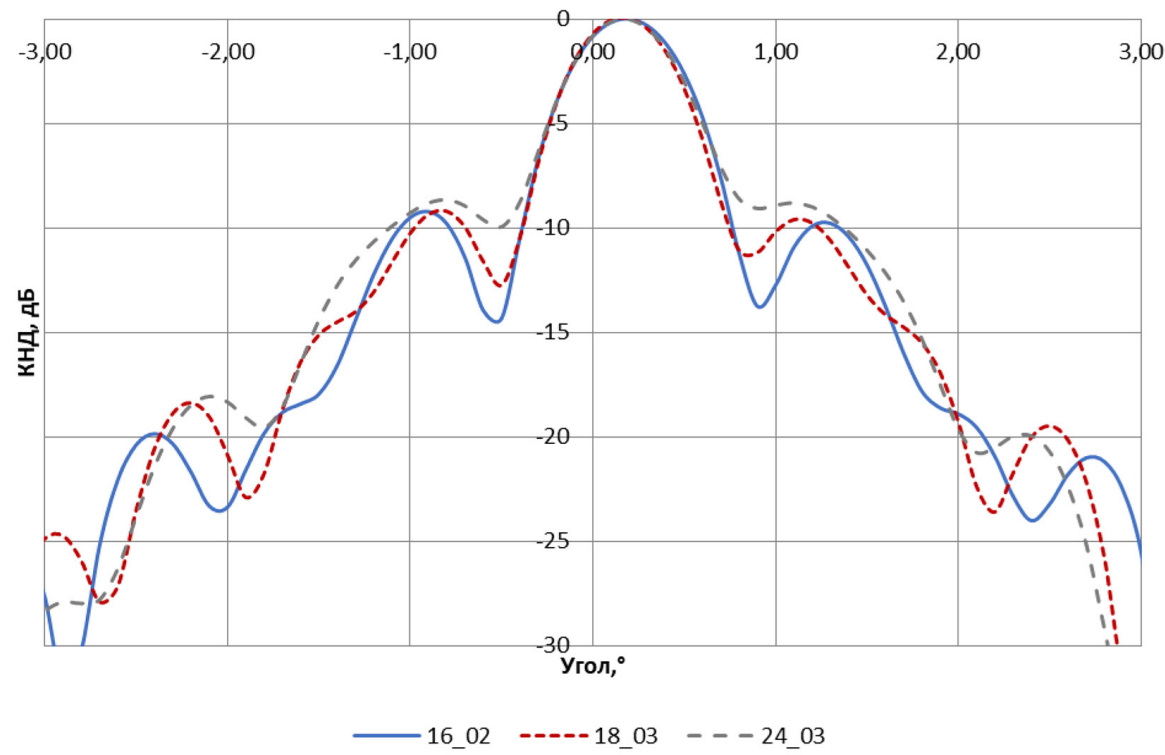

б)

Рис. 6. ДН антенны на частоте 43,924 ГГц: а - в плоскости $0^{\circ}$; 6 - в плоскости $90^{\circ}$

Fig. 6. Antenna pattern at a frequency of $43.924 \mathrm{GHz}: \mathrm{a}-$ in the plane of $0^{\circ} ; \mathrm{b}-$ in the plane $90^{\circ}$ 
Полученное значение КУ имеет крайне низкое значение, что идет вразрез с испытаниями антенны при работе через космический аппарат (КА), где полученные информационные скорости соответствуют более высокой энергетике.

Ширина ДН антенны больше ожидаемых значений (расчетное значение $0,4^{\circ}$ ), что может указывать на возможную причину низкого КНД по сравнению с расчетными значениями - облучающая система находится не в фокусе.

Отсутствие оптических систем контроля положения антенны делает невозможным анализ отклонения ДН от оси. Нельзя однозначно утверждать, вследствие чего произошло отклонение - из-за конструктивных особенностей антенны (отклонение облучающей системы от оси, влияние короба для оборудования и т. д.) или из-за наклона антенны на рабочем столе.

Таким образом, применение метода спирального сканирования позволяет за достаточно небольшое время получить реальные характеристики антенны и проводить анализ причин отклонения измеренных характеристик от рассчитанных.

\section{Благодарности / Acknowledgments}

Работа выполнена при финансовой поддержке Министерства науки и высшего образования Российской Федерации в ходе реализации комплексного проекта «Создание высокотехнологичного производства земных станций перспективных систем спутниковой связи для обеспечения связанности труднодоступных, северных и Арктических территорий Российской Федерации», осуществляемого при участии Сибирского федерального университета (соглашение № 075-11-2019-078 от 13.12.2019).

This work was carried out with the financial support of the Ministry of Science and Higher Education of the Russian Federation in the course of the implementation of the complex project «Creation of high-tech production of earth stations of promising satellite communication systems to ensure the connectivity of remote, northern and Arctic territories of the Russian Federation», carried out with the participation of the Siberian Federal University (agreement No. 075-11-2019-078 dated 12/13/2019).

\section{Список литературы / References}

[1] Выгонский Ю.Г., Кузовников А.В., Головков В.В., Сомов В.Г. Предложения по созданию космической системы для предоставления телематических услуг связи. Фундаментальные исследования, 2014, 9(4), 719-723 [Vygonskij YU.G., Kuzovnikov A. V., Golovkov V.V., Somov V. G. Proposals for the creation of a space system for the provision of telematic communication services. Basic research, 2014, 9(4), 719-723 (in Russian)]

[2] Андреев А.М., Богинский Л.П., Гришин М.В. Принщипы построения зарубежных спутниковых систем персональной подвижной связи. СПб.: ВКА имени А.Ф. Можайского, 2008, 345 c [Andreev A.M., Boginskij L.P., Grishin M.V. Principles of building foreign satellite systems for personal mobile communication, St. Petersburg.: VKA imeni A. F. Mozhajskogo, 2008, 345 p (in Russian)]

[3] Dmitriev D.D., Gladyshev A.B., Ratuschnyak V.N., Grithan O. B. Software and hardware complex for the development and research of methods for broadband access to multimedia resources and the Internet. Journal of Physics: Conference Series, 2020, 1515(3), 032041 p. 
[4] Захарьев Л.Н. Методы измерения характеристик антенн СВЧ. М.: Радио и связь, 1985, 368 c. [Zahar'ev L. N. Methods for measuring characteristics of microwave antennas. Moscow, Radio i svyaz', 1985, 368 p (in Russian)]

[5] Кирпанев А.В., Кирпанев Н.А. Принципы исследования антенн с обтекателем регулярной формы с помощью сферического сканера. Вопросы радиоэлектроники, 2020, 4, 14-21 [Kirpanev A. V., Kirpanev N. A. Principles of Studying Regular Radome Antennas Using a Spherical Scanner. Radio electronics issues, 2020, 4, 14-21(in Russian)]

[6] Испытательная система для измерения характеристик беспроводной связи R\&S TS8991[Электронный ресурс] - Режим доступа: https://www.rohde-schwarz.com/ru/product/ ts8991-productstartpage_63493-8444.html - Заглавие с экрана. [TS8991 OTA Performance Test System. Perfect characterization of wireless products over the air interface. [Electronic resourse] Access: https://www.rohde-schwarz.com/ru/product/ts8991-productstartpage_63493-8444.html 